\title{
The optimisation of shot peen forming processes
}

\author{
T. Wang ${ }^{* a}$, M.J. Platts ${ }^{\mathrm{b}}, \mathrm{J} . \mathrm{Wu}^{\mathrm{c}}$ \\ ${ }^{a}$ School of Engineering and Design, Brunel University, Uxbridge UB8 3PH, UK. \\ ${ }^{\mathrm{b}}$ Department of Engineering, University of Cambridge, Mill Lane, Cambridge, CB2 1RX, U.K. \\ ${ }^{\mathrm{c}}$ Department of Aircraft Manufacturing Engineering, Northwestern Polytechnical University, Xi'an, 710072, China
}

\begin{abstract}
An optimisation method based on an effective process model is presented. The calibrated and verified process model simulates peening on realistic components and provides a non-linear relationship between peening parameters and deflections. An optimal solution can be found by minimising the deviation of the computed deformed shape from the desired shape subject to certain constraints. This optimal solution of peening parameters can be directly used for practical operations. An optimisation example for forming a cylindrical shape on a $76 \times 76 \times 3 \mathrm{~mm}$ sample is provided. The experimental results demonstrate the applicability of the present optimisation method.
\end{abstract}

\section{Key words:}

Shot peening, (shot) peen forming, optimisation, process model, finite element analysis (FEA)

\section{Introduction}

Bombarding the surface of a metal sheet by a stream of small hard shot with sufficient kinetic energy can form a specific shape. This process is called (shot) peen forming, which has been especially used for contouring integral aircraft skin panels.

The key problem for peen forming processes is how to determine an optimal design of peening parameters for the desired shape. However, in practical applications this has been through trial and error. Tatton [1] described shot peen forming in production and emphasized the importance of experience. Some numerical methods that are heavily dependent upon tests were also developed. For example, VanLuchene and Cramer [2] presented a numerical method for predicting peening intensity patterns by assuming the peen forming process as a linear finite element system responding to the peening induced stresses. Empirical equations determined by physical experiments in Boeing Commercial Airplane Group were relied upon to set up the relationship between

\footnotetext{
* Corresponding author: Email address: tao.wang@brunel.ac.uk
} 
the induced stresses and the peening intensity. As recognized in their paper, Homer and VanLuchene [3] stated that the experimental data are based on peening of unconstrained specimens but assumed to be applicable for constrained specimens, which may introduce error because in reality various constraints, such as geometry, jigs (pre-stresses), and peening sequences, are possible.

Wang [4] and Wang et al. [5] presented a process model and verified it in various experimental conditions with different constraints. This model has also been discussed and further developed by Blackwell et al. [6] of QinetiQ. Using the calibrated and verified process model, a non-linear relationship between the peening parameters and deflection of specified points can be set up. A constrained optimisation procedure can consequently be used to find an optimal solution according to specified deflection. This optimisation method will be discussed in this paper.

\section{The process model}

Up to a certain intensity of coverage the individual peen impacts can be regarded as acting independently. A number of discrete impacts can therefore be assumed to act simultaneously and their effects are distributed in a specific plastic layer. Based on this assumption, a static loading unit is found to model the macroscopic effect of this shot peening. The loading unit can be conveniently calibrated from the peening parameters, such as shot radius, mass flow rate and air pressure. Realistic peening intensities can then be simulated by multiple application of this loading unit. The number of loading cycles is in direct proportion to the peening time, which makes the model applicable to practical applications. Each loading cycle includes two steps. In the first step, a temperature profile is applied to the section points through the thickness of composite shell elements in ABAQUS when all nodal degrees of freedom are fixed. This application of temperature causes the surface layer to be stretched beyond yield, thus mimicking the effect of peening. After the application of this equivalent load to induce a plastic layer, the second step is realised by simply releasing the extra boundary conditions. This leaves the material in a deformed configuration. More details of the model can be found in [4-5].

\section{The experimental approach}

$3 \mathrm{~mm}$ thick aluminium 5251-H22 sheet was used in the present study. Its proportional limit is about $103 \mathrm{MPa}$, Young's modulus 69GPa and Poisson's ratio 0.33. Uniaxial tension tests were conducted to provide the stressstrain relationship for the analysis. Cast steel S660 peens were employed in an air-blast machine. The mass flow rate was kept as a constant $13.67 \mathrm{~g} / \mathrm{s}$. The nozzle was kept vertical to the worktable at a distance of $300 \mathrm{~mm}$. 
Unconstrained 20psi peening on $76 \times 19 \times 3 \mathrm{~mm}$ samples was used for calibration tests. Only the central area $19 \times 19 \mathrm{~mm}$ was peened, with the rest protected by masking, because the distribution of peens within this area can be approximated as a uniform distribution for the peening machine being used. Arc height was measured by a digital Almen gauge like the normal Almen test.

As a particular example for the optimisation, a cylindrical shape on $76 \times 76 \times 3 \mathrm{~mm}$ samples with a curvature radius of $600 \mathrm{~mm}$ was desired under the experimental conditions. The cylindrical shape was chosen because it provides a challenge, as it is known that uniform unconstrained peening normally produces a double-curved shape. A digital dial gauge was used to measure the deformed shape on the peened surface. As the sample was not absolutely flat before peening, the measurement was also conducted before peening. The deflection was finally taken as the subtraction of the initial reading from the final one.

\section{The optimisation method}

\subsection{The assumption}

In a one-dimensional problem (one peening area and one deflection as the calibration test), it is found that a non-linear relationship

$$
w=\frac{a t}{b+t}
$$

between the peen-formed arc height $w$ and exposure time $t$ can fit both the experimental results and finite element analyses as shown in Fig.1.

For a multidimensional problem as shown in Fig.2, a similar relationship can be assumed as follows

$$
w_{i}=\frac{A_{i j} t_{j}}{B_{i j}+t_{j}} \quad(i=1,2, \ldots, n \text { and } j=1,2, \ldots, m)
$$

where $t_{j}$ is the exposure time (or a variable which is in direct proportion to it) on the $j$ th peening element only, $w_{i}$ the deflection of the $i$ th node, and $A_{i j}$ or $B_{i j}$ the non-linear fitting coefficients. This assumption implies that the deflection at the $i$ th node can be simply added by the components contributed by each peening element. This might involve certain errors because it is known that the peening sequence affects the deflection. However, as shown in [5], successively peening on two adjacent areas has a variation of about $5 \%$ which is neglectable.

As a convenient means, Eq.(2) can be written in the format of matrices

$$
\mathbf{K t}=\mathbf{w}
$$

or 


$$
\left[\begin{array}{cccc}
K_{11} & K_{12} & \ldots & K_{1 m} \\
K_{21} & K_{22} & \ldots & K_{2 m} \\
\ldots & \ldots & \ldots & \ldots \\
\ldots & \ldots & \ldots & \ldots \\
K_{n 1} & K_{n 2} & \ldots & K_{n m}
\end{array}\right]\left\{\begin{array}{c}
t_{1} \\
t_{2} \\
\ldots \\
t_{m}
\end{array}\right\}=\left\{\begin{array}{c}
w_{1} \\
w_{2} \\
\ldots \\
\ldots \\
w_{n}
\end{array}\right\}
$$

where $K_{i j}=\frac{w_{i}}{t_{j}}=\frac{A_{i j}}{B_{i j}+t_{j}}(i=1,2, \ldots, n$ and $j=1,2, \ldots, m)$. In the physical aspects, it means peening to one unit of $t_{j}$ on the $j$ th element can produce a deflection of $K_{i j}$ at the $i$ th node.

\subsection{The optimisation procedure}

For a given desired $\hat{\mathbf{w}}$, the solution to Eq.(3a) is generally not unique because $n$ might not be equal to $m$ and the components of $\hat{\mathbf{w}}$ might not be independent. Therefore, an optimisation procedure is needed to find an optimal solution $\overline{\mathbf{t}}$. As a forming process, the most important concern is the accuracy of the formed shape. The optimisation problem can be given as to find an optimal $\overline{\mathbf{t}}$ which minimises the target function

$$
f(\mathbf{t})=\|\mathbf{K} \mathbf{t}-\hat{\mathbf{w}}\|
$$

subject to specific constraints, for example,

$$
\left\{\begin{array}{c}
\mathbf{t}_{1} \leq \mathbf{t} \leq \mathbf{t}_{\mathbf{u}} \\
\|\mathbf{K t}-\hat{\mathbf{w}}\|_{\text {inf }} \leq \hat{\varepsilon}
\end{array}\right.
$$

where $\mathbf{t}_{1}$ and $\mathbf{t}_{u}$ are lower and upper bounds respectively, $\hat{\varepsilon}$ is the maximum allowed error. The optimisation procedure can be described as follows,

1) Discretise the 2D blank to peening elements and locate the specific nodes for assessing the deformed shape.

2) Determine the shot size and air pressure for the peening machine being used.

3) Conduct FE analysis on each of the peening elements to determine the individual $K_{i j}$

4) Assemble the overall matrix $\mathbf{K}$.

5) Determine the optimal $\overline{\mathbf{t}}$ subject to Eq.(4) and Eq.(5).

\section{Results and discussion}

For the purpose of forming a cylindrical shape on $76 \times 76 \times 3 \mathrm{~mm}$ samples with a curvature radius of $600 \mathrm{~mm}$, the $2 \mathrm{D}$ shape as shown in Fig. 3 is divided to 16 peening elements and 25 nodal points. Only one peening element 
was peened by masking others in each increment. The whole process was done by incrementally peening all 16 areas. The shot size and air pressure were set as stated above.

Process modelling was correspondingly conducted for a quarter of the model because the sample is symmetric. The computation result for modelling peening on a central area is shown in Fig.4. The overall matrix $\mathbf{K}$ is assembled according to all FEA results.

Referring to Fig.3, for a cylindrical shape with a curvature radius of $600 \mathrm{~mm}$, the component of the desired $\hat{\mathbf{w}}$ at point $\mathrm{A}$ and $\mathrm{E}$ is $0 \mathrm{~mm}$, point $\mathrm{B}$ and $\mathrm{D} 0.9 \mathrm{~mm}$, and point $\mathrm{C} 1.2 \mathrm{~mm}$ as shown in solid lines of Fig.5(a) (e) for sections 1 5. If $t_{l}$ and $t_{u}$ are set to 0 and 60 seconds and $\hat{\varepsilon}$ is $0.47 \mathrm{~mm}$, the optimal solution $\overline{\mathbf{t}}$ is shown in Fig.3 for each peening element. According to this optimal solution, the experimental results measured along section 1 5 at 25 points are shown in Fig.5. The deviation of the experimental results from the desired shape along sections 2, 3, and 4 is a few percent, while sections 1 and 5 have a relatively larger deviation. This could be attributed to the edge effects.

It is worth noting that $0.47 \mathrm{~mm}$ of $\hat{\varepsilon}$ is the minimum error that the optimisation procedure can achieve for this tested case. If $\hat{\varepsilon}$ is set to a smaller value, the constraints given by Eq. (5) cannot be satisfied. This indicates that a slight double-curved result cannot be avoided by this unconstrained peening and pre-stressed peening could be further used to shape a more single-curved shape.

\section{Conclusion}

As demonstrated in the present work, the optimisation method based on the effective process model is able to provide a preliminary optimal design of peening parameters for the desired shape. The process model serves like virtual tests on realistic components and can produce a more accurate simulation than previous models. From the modelling results, a non-linear relationship between peening parameters specified for discrete peening areas and deflections specified for nodal points can be set up. On the basis of this relationship, a constrained optimisation procedure can be used to find an optimal solution which can be directly used for practical operations.

\section{Acknowledgement}

This paper is based on a project initially carried out in Cambridge University Engineering Department, which was supported by Airbus UK, Cambridge Overseas Trust, and Universities UK. 


\section{References:}

[1] Tatton, R.J.D., Shot peen forming, in Impact Surface Treatment (The Second International Conference on Impact Treatment Processes, Cranfield Institute of Technology, Bedford, UK), S.A. Meguid, Editor. 1986, Elsevier: London. p. 134-143.

[2] VanLuchene, R.D. and Cramer, E.J., Numerical modelling of a wing skin peen forming process. Journal of Materials Engineering and Performance, 1996. 5(6): p. 753-760.

[3] Homer, S.E. and VanLuchene, R.D., Aircraft Wing skin contouring by shot peening. Journal of Material Shaping Technology, 1991. 9(2): p. 89-101.

[4] Wang, T., Numerical simulation and optimisation of shot peen forming processes, PhD Thesis, University of Cambridge, 2003.

[5] Wang, T., Platts, M.J, and Levers, A., A process model for shot peen forming. Journal of Materials Processing Technology, 2006, 172(2): p. 159-162.

[6] Blackwell, P.L., Griffiths, M.D., Ward, T. and Gardiner, S., A Computer Modelling Capability for Shot Peen Forming, Metal Finishing News, vol. 5, 2004. 
Figures

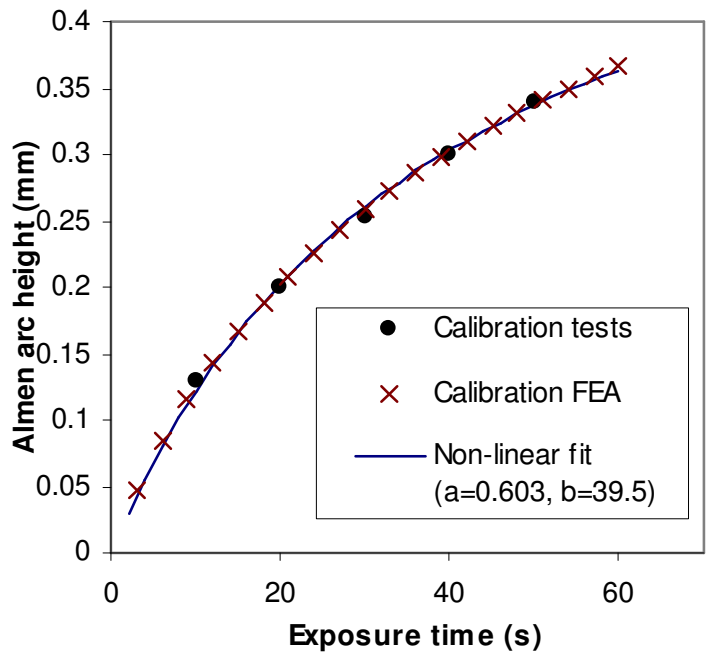

Figure 1 The deflection development of a one-dimensional problem

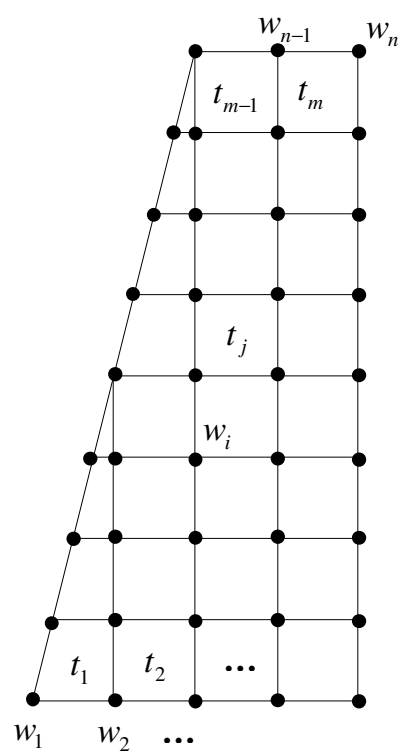

Figure 2 The configuration for a multidimensional problem 


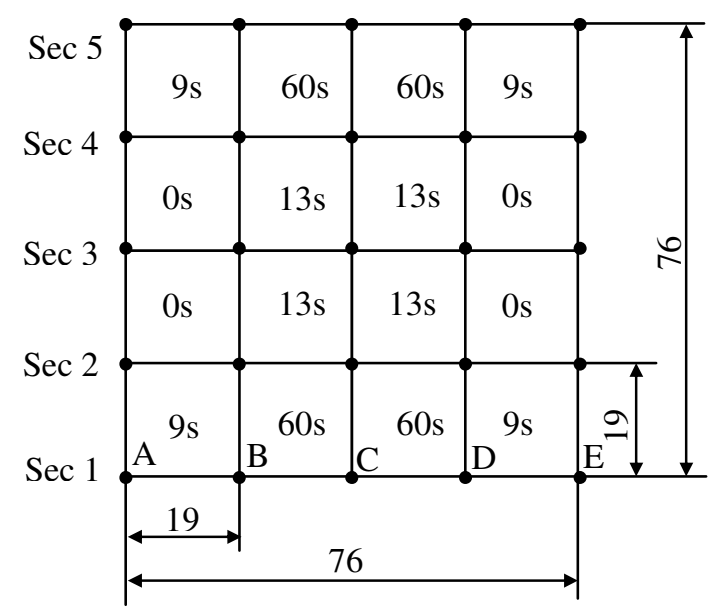

Figure 3 The configuration for an optimisation example

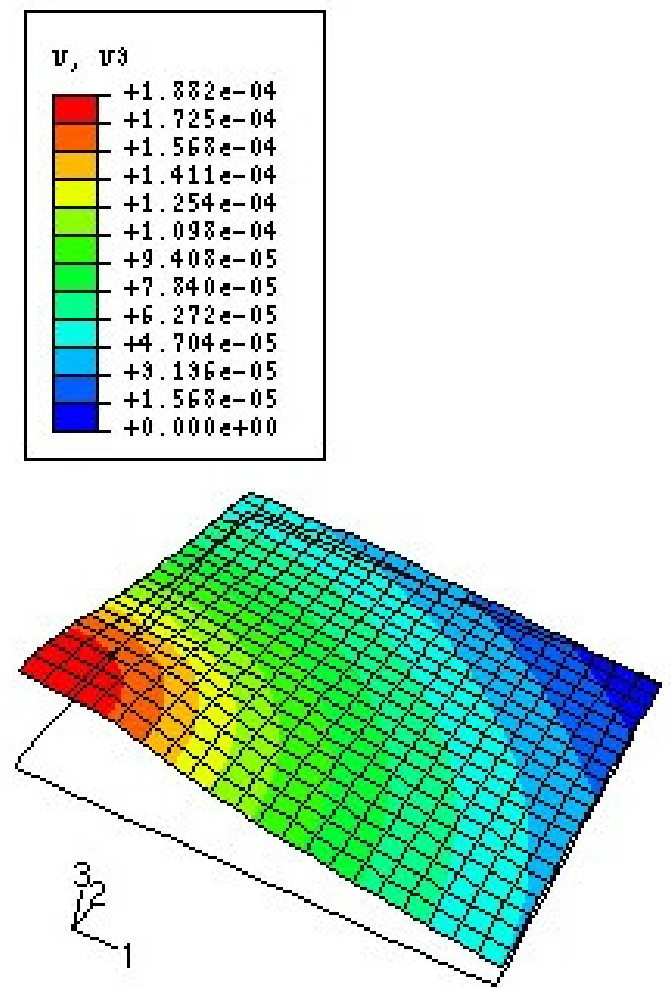

Figure 4 The computation result for modelling shot peening on a central area 


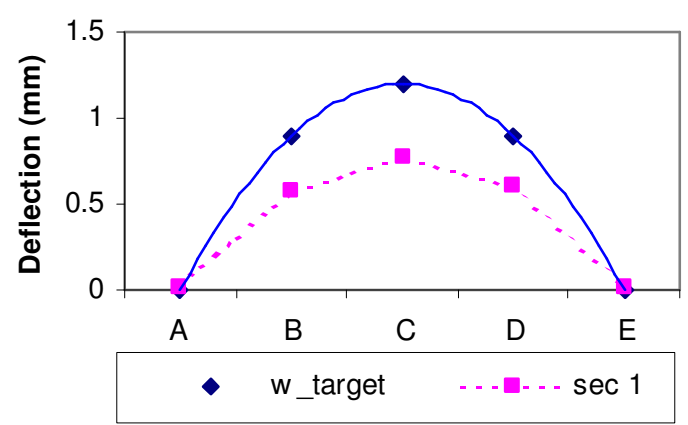

(a)

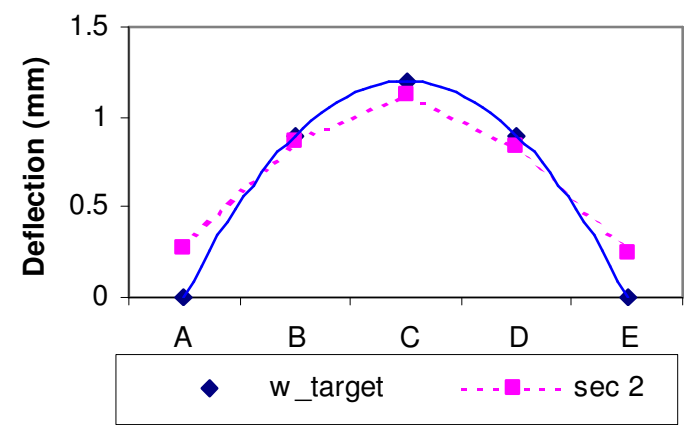

(b)

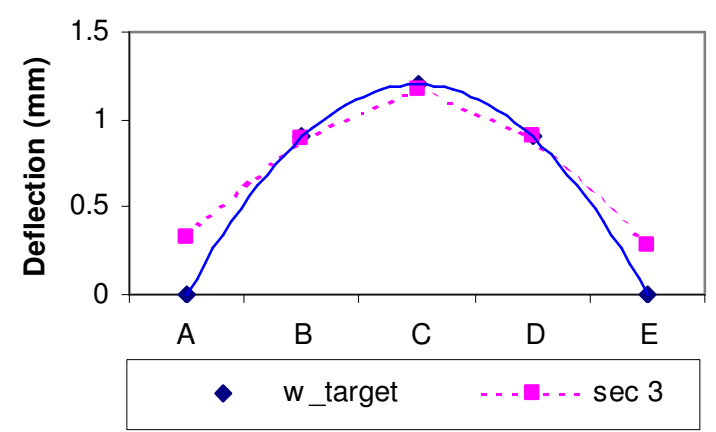

(c)

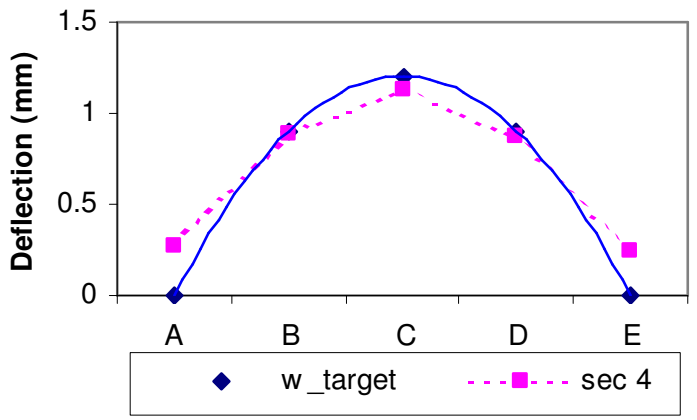

(d) 


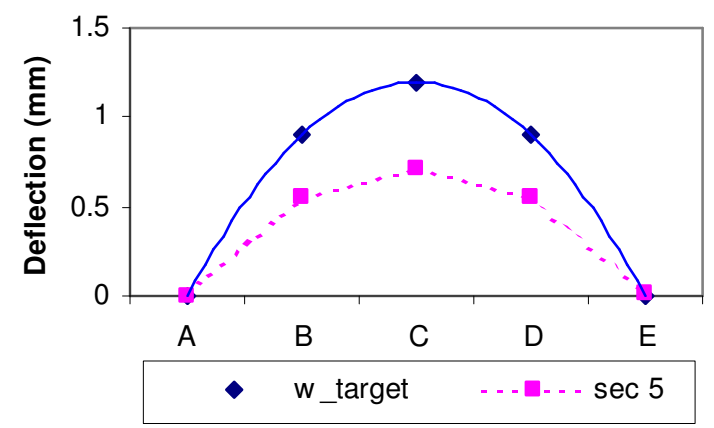

(e)

Figure 5 The comparison of the desired shape and the experimental results 